\title{
Nrf2 transcriptional derepression from Keap1 by dietary polyphenols
}

(Running title: Polyphenols derepress Nrf2 inhibition by Keap1)

\author{
Henry K. Bayele, ${ }^{* 1}$ Edward S. Debnam, ${ }^{2}$ and Kaila S. Srai ${ }^{1}$ \\ ${ }^{1}$ Department of Structural \& Molecular Biology, Division of Biosciences, University College \\ London, Gower Street, London WC1E 6BT, United Kingdom \\ ${ }^{2}$ Department of Neuroscience, Physiology, and Pharmacology, University College London, \\ Royal Free Campus, Rowland Hill Street, London NW3 2PF, United Kingdom
}

* Corresponding author: Department of Structural \& Molecular Biology, University College

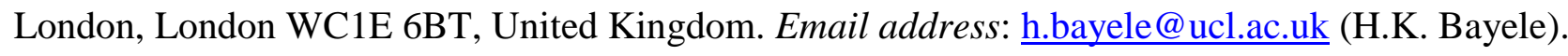

Total word count: $\quad 4,588 ; \quad$ Number of figures: 4

Abbreviations: ARE, antioxidant response element; hQR1, human NADP(H):quinone reductase 1; GST-Ya, Glutathione S-transferase Ya subunit; Nrf2, NFE-E2-related factor 2; Keap1, Kelch-like ECH-associated protein 1; t-BHQ, tert-butyl hydroquinone; DMSO, dimethylsuphoxide. 


\begin{abstract}
The liver expresses batteries of cytoprotective genes that confer cellular resistance to oxidative stress and xenobiotic toxins, and protection against cancer and other stress-related diseases. These genes are mainly regulated by Nrf2, making this transcription factor a target for small molecule discovery to treat such diseases. In this report, we identified dietary polyphenolic antioxidants that not only activated these genes but also relieved Nrf2 repression by Keap1, a Cul3dependent ubiquitin ligase adaptor protein that mediates its degradation. Analysis of postprandial liver RNA revealed a marked activation of both genes by all test polyphenols compared with controls. Nrf2 inhibition by RNA interference reduced polyphenol effects on its target gene expression. Our data suggest that polyphenols may induce cellular defense genes by derepressing Nrf2 inhibition by Keap1. We posit that this ability to derepress Nrf2 and reactivate its target genes may underlie the protection conferred by polyphenols against oxidative stress-related diseases.
\end{abstract}

Keywords: Antioxidant; antioxidant response element; detoxification; dietary polyphenols; cytoprotection.

\title{
1. Introduction
}

Oxidative and xenobiotic stresses cause a range of diseases through the production of reactive oxygen species. In response to these stresses, the liver expresses multidinous cytoprotective proteins most of which act as antioxidants or detoxification systems [1]. Archetypal enzymes/genes of this stress-response pathway include GST, QR1, and $\gamma$-glutamylcysteine synthetase. In response to a spectrum of chemical agents including diphenols, quinones, isothiocyanates and heavy metals, these genes are activated by Nrf2 [1, 2]. While endogenous antioxidant systems e.g. thioredoxin can counter redox stress, there is strong evidence that dietary polyphenols also modify the redox stress responses through Nrf2, possibly in synergy with the aryl hydrocarbon receptor [1, 3]. These 
compounds are therefore of immense interest as therapeutics against cancer, cardiovascular and inflammatory diseases [4].

The current working model of Nrf2 post-translational redox regulation posits that under basal (unstressed) conditions, it is sequestered in the cytoplasm by Keap1, a Cul3-dependent ubiquitin ligase adaptor protein that targets Nrf2 for ubiquitination and proteasomal degradation [5]. Oxidative stress and electrophiles inhibit this process allowing Nrf2 to translocate to the nucleus where it dimerizes with the small Maf proteins; the heterodimers then bind to the antioxidant response elements (AREs) of Nrf2 target genes to initiate their transcription. The core sequence of the ARE is TGACnnnGC (where $\mathrm{n}$ is any nucleotide); mutations within the TGAC and/or GC boxes attenuate antioxidant-dependent gene expression $[\mathbf{2 , 6 ]}$. The ARE is also found in genes involved in processes as diverse as iron metabolism, inflammation, and wound healing [7-10]. These genes synergistically constitute the main defences against both biotic and abiotic stresses. Consequently, Nrf2 knock-out mice are unable to resist oxidative or xenobiotic stress or detoxify drugs, and are susceptible to carcinogenesis and autoimmune inflammatory diseases [11-13]. However, mutations in Keap1 that reduce its Nrf2-binding capacity and repressivity can also lead (paradoxically) to disease through unrestrained Nrf2 expression [14-17]. Keap1 overexpression has also been associated with poor prognosis in renal cell carcinoma; increased expression thioredoxin improved prognosis [18]. This strongly suggests that constitutive repression of Nrf2 by Keap1 may lead to diminished cytoprotection against the disease. Thus inducing a redox shift in favour of Nrf2 derepression from Keap1 could conceptually reactivate Nrf2-target genes to prevent disease. This provided the rationale for this study: to identify small molecules that would reactivate Nrf2-target genes that are repressed by Keap1. We screened a small library of dietary polyphenols for their potential to: (a) induce Nrf2-target gene expression and, (b) derepress these genes when they are transcriptionally-silenced by Keap1. Our findings indicate that the ability of polyphenols to protect against disease may be coupled to their ability to derepress Nrf2 from Keap1. 


\section{Materials and methods}

\section{$2.1 \quad$ Reagents.}

2.2 A small library of polyphenols (Table 1) was purchased from Sigma (Poole, UK). This included quercetin, kaempferol, caffeic acid, rutin, resveratrol, ferulic acid, luteolin, naringenin, epicatechin, (-)-epigallocatechin-3-gallate (EGCG), sulforaphane and tert butylhydroquinone. The compounds were dissolved in DMSO as $100 \mathrm{mM}$ stock solutions. All other analytical grade chemicals were also obtained from Sigma.

\subsection{Plasmid constructs.}

The human $Q R 1$ promoter (750bp) was amplified from human genomic DNA with the primers: CATGCTAGCACCTGCCTTGAGGAGCAGG (hQR1P-S)

and CATGCTCGAGAGCACTCACCGACCATGG (hQR1P-A). Primers were derived from Accession number M81596 [19]. The PCR product was restricted with NheI and XhoI (sites underlined), and ligated into the same sites in pGL3Basic vector (Promega) to generate hQR1Pluc. The mutant promoter vector $h \mathrm{QR} 1 \mathrm{Pluc} \triangle \mathrm{ARE}$ lacking the $\mathrm{ARE}$, was generated by site-directed mutagenesis with the QuikChange mutagenesis kit (Stratagene).

For ARE-luciferase enhancer constructs, the following phosphorylated primers containing the AREs (bold-face) were synthesized (MWG Biotech): rQR1-ARE sense, CTAGCGAATTCAGTCTAGAGTCACAGTGACTTGGCAAAATCTGAC; rGST-Ya ARE sense,

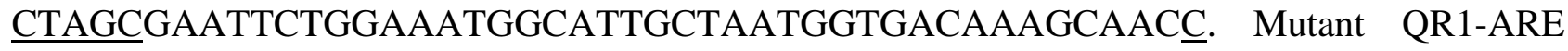
oligonucleotides were also synthesized as follows: AREMt sense, CTAGCGAATTCAGTCTAGAGTCACAGTaACTTGaCAAAATCTGAC. Oligonucleotides were annealed [20] and ligated directionally into the NheI-XhoI sites of the pGL3 Promoter vector (Promega). Recombinant plasmids were verified by linearization at unique EcoRI sites within the oligonucleotides. The resulting constructs were labeled QR-ARELuc, GST-ARELuc and AREMtLuc respectively. All plasmids were sequenced (MWG Biotech) for verification. 


\subsection{Cell culture, transfection and reporter assays.}

HepG2 cells (ECACC, UK) were cultured in $10 \mathrm{~cm}$ dishes in Minimum Essential Medium with Earle's salts supplemented with non-essential amino acids, L-glutamine, 10\% FBS, antibiotics and antimycotics (Invitrogen). Cells were transfected at $80 \%$ confluence with $10 \mu \mathrm{g}$ of ARE-Luc constructs and $1 \mu \mathrm{g}$ pSV $\beta$ gal (Promega) as internal control, using FuGENE 6 (Roche) according to the manufacturer's instructions. After $18 \mathrm{~h}$, the cells were trypsinized, centrifuged, resuspended in fresh medium and distributed into 24-well plates (Corning); they were treated with various polyphenols or with DMSO (solvent control) for $24 \mathrm{~h}$. For transactivation or repression, cells were seeded in 24-well plates and cotransfected with $100 \mathrm{ng}$ ARE-Luc constructs and $100 \mathrm{ng}$ Nrf2 alone or together with $100 \mathrm{ng}$ Keap1 expression plasmids respectively; $50 \mathrm{ng}$ pSVßgal was included as internal control. The cells were then treated with DMSO or with selected polyphenols for $24 \mathrm{~h}$. Analysis of reporter gene expression was as previously described [20].

\subsection{Nrf2 knock-down.}

A retroviral vector (pRS-hNrf2) expressing short-hairpin RNA to human Nrf2 was used to transfect Hep3B cells with $5 \mu \mathrm{g} / 10 \mathrm{~cm}$ dish using Fugene HD (Roche). In parallel, cells were transfected with the negative control vector pRS-GFP. After $48 \mathrm{~h}$, the medium was replaced and supplemented with $2 \mu \mathrm{g} / \mathrm{ml}$ puromycin (Sigma). Stable cell lines were established after 3 weeks of continuous selection. Both cell lines were transfected with hQR1Pluc and pSVßgal, and then treated with polyphenols as described above.

\subsection{Expression and purification of recombinant Nrf2 and MafG.}

pET15b-Nrf2 and pET15b-MafG plasmids were transformed into BL21-CodonPlus (DE3)RIPL cells (Stratagene) and induced with $0.4 \mathrm{mM}$ IPTG for $3 \mathrm{~h}$ at $37^{\circ} \mathrm{C}$. Recombinant His-Nrf2 and His-MafG were purified using the MagneHis Protein Purification kit (Promega) as recommended by the manufacturer. 


\subsection{Analysis of phase II gene activation in vivo by polyphenols.}

Animals were handled according to UCL institutional guidelines approved by the UK Home Office. Male Sprague-Dawley rats (230-290 g), maintained ad libidum on standard rat chow (Diet RM1, United Kingdom), were gavage-fed with $50 \mathrm{mg} / \mathrm{kg}$ body weight of kaempferol, naringenin, quercetin and resveratrol dissolved in $1 \mathrm{ml}$ of $10 \% \mathrm{DMSO} / 50 \%$ ethanol in normal saline; control animals were gavage-fed with $1 \mathrm{ml}$ of carrier solvent. After $18 \mathrm{~h}$, the rats were anaesthetised with pentobarbitone sodium (90 mg/kg, i.p.) and the livers were removed into RNAlater (Ambion). Total RNA was extracted using the Qiagen RNeasy kit (Qiagen). Two parallel Northern blots of RNA (10 $\mu \mathrm{g}$ ) resolved on a $1 \%$ formaldehyde gel and transferred onto Hybond $\mathrm{N}+$ membrane (Amersham Biosciences) were pre-hybridized in UltraHyb buffer (Ambion) for $30 \mathrm{~min}$ at $42^{\circ} \mathrm{C}$. The blots were then hybridized overnight in the same buffer at $42^{\circ} \mathrm{C}$ with complementary oligonucleotide probes to rat $Q R 1$ (CTCTCTGCGTGGGCCAATACAATCAGGGCTCTTCTCACCGCCAT) or rat GST-Ya (TGCCCCGGGCATTGAAGTAGTGAAGCACTGGCTTCCCAGACAT), end-labeled with $[\gamma$ -

$\left.{ }^{32} \mathrm{P}\right]$ ATP. Post-hybridization washes were performed with $3 \mathrm{X} 15 \mathrm{~min}$ in SSC/0.1\% SDS and then for $30 \mathrm{~min}$ at $60^{\circ} \mathrm{C}$ in $0.1 \mathrm{XSSC} / 0.1 \% \mathrm{SDS}$. The blots were exposed to Kodak X-omat film (Sigma) overnight at $-80{ }^{\circ} \mathrm{C}$.

\subsection{Electrophoretic mobility shift assay (EMSA).}

HepG2 cells were treated with $100 \mu \mathrm{M}$ selected polyphenols or with DMSO $(0.1 \%$ final concentration) for $6 \mathrm{~h}$. Nuclear extracts were prepared and mobility shift assays were performed as previously described [20], using duplex ARE oligonucleotides (described above) end-labeled with $\gamma$-32P[ATP]. For competition, unlabeled probe was added in molar excess (100 pmols) to the extract for $10 \mathrm{~min}$ before adding the radiolabeled probe. To confirm binding specificity, His-Nrf2 and His-MafG were incubated with the ARE probes as homodimers or heterodimers. Binding reactions were resolved and processed as described previously [20]. 


\subsection{Western blotting.}

Nuclear extracts were prepared from the livers of rats treated as above. Approximately 10 $\mu \mathrm{g}$ of the extracts were resolved on 4-12\% NUPAGE Novex Bis-Tris gels using MES-SDS running buffer (Invitrogen). Proteins were and transferred to Immobilon-P membranes (Millipore). The blot was blocked for $1 \mathrm{~h}$ at room temperature in Tris-buffered saline/0.5\% Tween 20 (TBST) containing $5 \%$ non-fat milk, and then incubated at $4{ }^{\circ} \mathrm{C}$ overnight with anti-Nrf2 antibody (R \& D Systems; cat \# MAB3925). The membrane was washed 3X 5 min with TBST and incubated with goat anti-rabbit IgG HRP-conjugate (Sigma). Nrf2 was detected using the Visualizer Western blotting kit (Millipore) in a LAS-1000 Image Reader (Fujifilm) set for chemiluminescence detection. The membrane was stripped and re-probed with an HRP-conjugated anti-actin antibody (Abcam) to ascertain equivalent protein loading.

\subsection{Chromatin immunoprecipitation assay.}

To determine Nrf2 recruitment to the $h Q R 1$ promoter within its genomic context, HepG2 cells were either treated with either DMSO or with polyphenols for 6 h. Following formaldehyde cross-linking, chromatin was isolated and precipitated with $10 \mu \mathrm{g}$ of the above Nrf2 antibody, using a chromatin immunoprecipitation assay kit (Upstate Biotechnology) as instructed by the manufacturer. The immune complexes were eluted and DNA was purified by phenol-chloroform extraction and ethanol-precipitation as previously described [20]. For PCR, primers hQR1P-S (above) and GGCTGCAGCTCCAGGAAGGGAG were used to amplify a 500bp fragment of the hQR1 promoter over 35 cycles; PCR products were resolved on a $2 \%$ agarose gel in $1 \mathrm{X}$ TAE.

\subsection{Statistical analysis.}

Data analyses were performed and graphs were plotted with the GraphPad Prism software (GraphPad Software). Statistical significance was determined by the Mann-Whitney U test or the 
one-way analysis of variance (ANOVA)/Student-Newman-Keuls multiple-comparisons test where applicable. Probability $(\mathrm{P})$ values $<0.05$ were accepted as significant.

\section{Results}

\subsection{Differential activation of phase 2 genes from cognate AREs by polyphenols.}

We set out to identify natural small molecule Nrf2 activators that would induce cytoprotective genes. Reporter vectors of the cognate AREs (and mutants) of two archetypal cytoprotective genes $Q R I$ and GST-Ya (Fig. 1A) were transfected into HepG2 cells which were then treated with a selection of dietary polyphenols or with DMSO. Reporter assays showed that the AREs were functionally sufficient in driving basal luciferase expression. Treatment of the transfected cells with various polyphenols showed that compared with DMSO, these compounds enhanced luciferase expression several-fold from wild-type but not from mutant ARE constructs (Fig. 1B, C). Based on these data, we could stratify these compounds by the level to which they could induce ARE-mediated luciferase expression. Potent inducers were the flavonols quercetin, naringenin and kaempferol, while the tea-derived catechin EGCG, which belongs to the flavan group of polyphenols, was a poor inducer. While both core ARE elements in the enhancer constructs are conserved the activation profiles for both $Q R I$ and $G S T-Y a$ were similar but not identical; they elicited some differences in luciferase expression suggesting that sequences juxtaposing the cognate core AREs may be important [10]. This was manifested by Nrf2 coexpression with the cognate AREs (Fig. 1D). To test the ARE in a larger genomic context, we cloned $\sim 750 \mathrm{bp}$ of the human $Q R 1$ promoter; the ARE was mutated by site-directed mutagenesis. Luciferase assays revealed that Nrf2 transactivated wild-type full-length hQR1luc but not its ARE mutant (Fig. 1E), confirming the crucial role of the ARE in phase II gene expression. 


\subsection{Transcriptional derepression of Nrf2 from Keap1 by polyphenols.}

To establish whether polyphenols could reactivate Keap1-silenced genes, we first cotransfected hQR1luc with increasing concentrations of Keap1 into HepG2 cells; we found a dosedependent repression of Nrf2 transactivation of promoter activity by Keap1 (Fig. 2A). Intriguingly, this repression was completely reversed by polyphenol treatment (Fig. 2B). In similar experiments with the ARE enhancer constructs, we observed that Keap1 co-expression reduced ARE transactivation by Nrf2 but this was also completely reversed when cells were treated with polyphenols (Fig. 2C, D). This suggests that polyphenols may confer cytoprotection by Nrf2 transcriptional derepression from Keap1. To further confirm the role of Nrf2 in polyphenoldependent gene activation, we generated a hepatoma cell line that constitutively expressed short hairpin RNAs that target Nrf2. Transfection of these cells with hQR1luc and treatment with polyphenols revealed a $60 \%$ reduction in ARE responsiveness compared with cells expressing control short hairpin RNA against GFP (Fig. 2E).

\subsection{Recruitment of Nrf2 to AREs in vitro and in vivo.}

To further confirm that Nrf2 was induced and recruited to the AREs by polyphenol treatment, we performed EMSA. Nuclear extracts were prepared from HepG2 cells treated with a selection of polyphenols while control cells were treated with DMSO. Mobility shift showed that while the mutant ARE was unable to bind, we found specific binding to wild-type AREs by nuclear proteins from polyphenol-treated cells compared with DMSO-treated cells (Fig. 3A, B). Specific binding was ascertained by inclusion of a 100-fold molar excess of the cold ARE probe; this showed competitive inhibition of binding by radiolabeled ARE. EMSA with recombinant Nrf2 and MafG also showed that MafG-Nrf2 heterodimers bound strongly to the ARE; this binding showed Nrf2 concentration-dependence (Fig. 3C, D). Finally, ChIP assays with an Nrf2-specific antibody showed that compared with DMSO, polyphenols induced Nrf2 recruitment to the AREs in HepG2 cells (Fig. 3E). 


\subsection{In vivo induction of phase 2 genes by polyphenols.}

In order to study the in vivo effects of polyphenols on phase II gene expression, age-matched rats were gavage-fed for $18 \mathrm{~h}$ with kaempferol, naringenin, quercetin and resveratrol; control animals were gavage-fed with DMSO in normal saline. Northern blotting of liver RNA revealed a marked up-regulation in both $Q R 1$ and $G S T-Y a$ expression in rats fed with polyphenols compared with DMSO-fed controls (Fig. 4A). Densitometric scans of $Q R 1$ and $G S T$ - $Y a$ transcripts on the blot showed differences in gene expression induced by the polyphenols; their potencies were in the order: quercetin > resveratrol > naringenin > kaempferol. To test whether these compounds induced Nrf2 in vivo, we performed Western blotting on rat liver cell nuclear extracts. We found increased Nrf2 protein levels, with resveratrol and quercetin being better inducers than naringenin and kaempferol (Fig. 4B).

\section{Discussion}

Our study was predicated on finding natural small molecules that could modify the dynamics of Nrf2-Keap1 interaction. We focused on dietary polyphenols because although several studies have supported their involvement in cellular defense against oxidative stress [21], the molecular basis of their chemoprotective potential is only partially understood. We chose $Q R I$ and GST-Ya as exemplars of Nrf2 target genes because they have been shown to be integral to body defenses against environmental toxins, oxidative stress-induced cell death and carcinogenesis [22, 23]. We therefore developed a surrogate system using the AREs of $Q R I$ and GST-Ya fused to the luciferase gene. Reporter assays showed that polyphenols differ in their abilities to induce gene expression. The flavonols (e.g. kaempferol) were potent in transcriptional activation of AREdependent reporter expression while the tea flavan EGCG was not active in vitro. This therefore suggests that polyphenol stereochemistry may determine their relative antioxidant potentials and therefore their capacity for Nrf2-target gene activation. These potencies were reflected in post- 
prandial liver RNA analysis which showed that rats fed polyphenols showed differential phase II gene induction; compared with DMSO, all the compounds tested increased $Q R I$ and GST-Ya mRNA levels, with quercetin inducing the highest expression levels.

Although largely regarded as antioxidants, most polyphenols have (residual) pro-oxidant activity due to their ability to generate free hydroxyl radicals (oxidative stress) especially in the presence of transition metals including copper and iron [24]. Oxidative stress and electrophiles induce cysteine modifications in Keap1, enabling Nrf2 to activate its battery of defense genes [2, 25]. Our experiments were therefore designed to assess how dietary polyphenols affect the Nrf2Keap1 dynamics. We found up-regulation of ARE-luciferase activity with Nrf2 while Nrf2 knockdown or ARE deletion reduced gene inducibility by polyphenols. These experiments suggest that the ARE might serve as a regulatory node for Nrf2-target gene repression-derepression cycles. Although Keap1 repressed Nrf2, this repression could be completely reversed by polyphenols suggesting that these compounds may modify the dynamics of the Nrf2-Keap1 interaction in vivo to ensure cytoprotective gene transcription. Thus we envision a mechanism where the proportion of transcriptionally active Nrf2-target genes could be determined by diet. Our findings therefore improve our understanding of how polyphenols may differentially affect gene expression and human health through their ability to derepress Nrf2 and its target genes that are silenced by Keap1. We suggest that this ability may underlie the "French paradox", i.e. the low incidence of coronary heart disease in some regions (e.g. Mediterranean) despite a relatively high intake of foods rich in saturated fat [26]. In those regions, diets are rich in fruits and vegetables, indicating that polyphenol-rich diets may have a direct role in disease prevention. However it is also speculated that the French paradox may be attributed to the ability of some polyphenols such as resveratrol to mimic the oestrogen receptor (ER) ligand oestradiol and to activate ER signaling [27].

\section{Financial support}

H.K.B is supported by a Charles Wolfson Trust Senior Research Fellowship. 


\section{Acknowledgments}

We thank Cecil Pickett and M. Yamamoto for Nrf2 and Keap1 mammalian expression plasmids respectively. We are also grateful to John Hayes for pET15-Nrf2 and pET15-MafG, and to Roland Wolf for the short-hairpin RNA interference vectors pRS-GFP and pRS-hNrf2. We thank Joanne Marks for help with the animal work.

\section{References}

[1] T. Prestera, W.D. Holtzclaw, Y. Zhang, P. Talalay. Chemical and molecular regulation of enzymes that detoxify carcinogens. Proc. Natl. Acad. Sci. USA 90 (1993) 2965-2969.

[2] T. Nguyen, P.J. Sherratt, C.B. Pickett. Regulatory mechanisms controlling gene expression mediated by the antioxidant response element. Annu. Rev. Pharmacol. Toxicol. 43 (2003) 233-260.

[3] H.P. Ciolino, P.J. Daschner, G.C. Yeh. Dietary flavonols quercetin and kaempferol are ligands of the aryl hydrocarbon receptor that affect CYP1A1 transcription differentially. Biochem. J. 340 (1999) 715-722.

[4] E. Middleton Jr, C. Kandaswami, T.C. Theoharides. The effects of plant flavonoids on mammalian cells: implications for inflammation, heart disease, and cancer. Pharmacol. Rev. 52 (2000) 673-751.

[5] A. Kobayashi, M.I. Kang, H. Okawa, M. Ohtsuji, Y. Zenke, T. Chiba, K. Igarashi, M. Yamamoto. Oxidative stress sensor Keap1 functions as an adaptor for Cul3-based E3 ligase to regulate proteasomal degradation of Nrf2. Mol. Cell. Biol. 24 (2004) 7130-7139.

[6] W.W. Wasserman, W.E. Fahl. Functional antioxidant responsive elements. Proc. Natl. Acad. Sci. USA 94 (1997) 5361-5366. 
[7] E.C. Pietsch, J.Y. Chan, F.M. Torti, S.V. Torti. Nrf2 mediates the induction of ferritin H in response to xenobiotics and cancer chemopreventive dithiolethiones. J. Biol. Chem. 278 (2003) 2361-2369.

[8] J. Alam, D. Stewart, C. Touchard, S. Boinapally, A.M.K. Choi, J.L. Cook. Nrf2, a Cap'n'Collar transcription factor, regulates induction of heme oxygenase-1 gene. J. Biol. Chem. 274 (1999) 26071-26078.

[9] K. Itoh, M. Mochizuki, Y. Ishii, T. Ishii, T. Shibata, Y. Kawamoto, V. Kelly, K. Sekizawa, K. Uchida, M. Yamamoto. Transcription factor Nrf2 regulates inflammation by mediating the effect of 15-deoxy-delta(12,14)-prostaglandin j(2). Mol. Cell. Biol. 24 (2004) 36-45.

[10] S. Braun, C. Hanselmann, M.G. Gassmann, U. auf dem Keller, C. Born-Berclaz, K. Chan, Y.W. Kan, S. Werner. Nrf2 transcription factor, a novel target of keratinocyte growth factor action which regulates gene expression and inflammation in the healing skin wound. Mol. Cell. Biol. 22 (2002) 5492-5505.

[11] J. Li, T.D. Stein, J.A. Johnson. Genetic dissection of systemic autoimmune disease in Nrf2deficient mice. Physiol. Genomics 18 (2004) 261-272.

[12] M. Ramos-Gomez, M.-K. Kwak, P.M. Dolan, K. Itoh, M. Yamamoto, P. Talalay, T.W. Kensler. Sensitivity in carcinogenesis is increased in chemoprotective efficacy of enzyme inducers is lost in $n r f 2$ transcription factor-deficient mice. Proc. Natl. Acad. Sci. USA 98 (2001) 3410-3415.

[13] L. Leung, M. Kwong, S. Hou, C. Lee, J.Y. Chan. Deficiency of the Nrf1 and Nrf2 transcription factors results in early embryonic lethality and severe oxidative stress. J. Biol. Chem. 278 (2003) $48021-48029$.

[14] T. Shibata, A. Kokubu, M. Gotoh, H. Ojima, T. Ohta, M. Yamamoto, S. Hirohashi. Genetic alteration of Keapl confers constitutive Nrf2 activation and resistance to chemotherapy in gallbladder cancer. Gastroenterology 135 (2008) 1358-1368. 
[15] T. Shibata, T. Ohta, K.I. Tong, A. Kokubu, R. Odogawa, K. Tsuta, H. Asamura, M. Yamamoto, $\mathrm{S}$. Hirohashi. Cancer related mutations in NRF2 impair its recognition by Keap1-Cul3 E3 ligase and promote malignancy. Proc. Natl. Acad. Sci. USA 105 (2008) $13568-13573$.

[16] P. Nioi, T. Nguyen. A mutation of Keap1 found in breast cancer impairs its ability to repress Nrf2 activity. Biochem. Biophys. Res. Commun. 362 (2007) 816-821

[17] J.D. Hayes, M. McMahon. NRF2 and KEAP1 mutations: permanent activation of an adaptive response in cancer. Trends Biochem. Sci. 34 (2009) 176-188

[18] H. Ronkainen, M.H. Vaarala, S. Kauppila, Y. Soini, T.K. Paavonen, J. Rask, P. Hirvikoski. Increased BTB-Kelch type substrate adaptor protein immunoreactivity associates with advanced stage and poor differentiation in renal cell carcinoma. Oncol. Rep. 21 (2009) $1519-1523$.

[19] A.K. Jaiswal. Human NAD(P)H:quinone oxidoreductase (NQO1) gene structure and induction by dioxin. Biochemistry 30 (1991) 10647-10653.

[20] H.K. Bayele, H. McArdle, S.K.S. Srai. Cis and trans regulation of hepcidin expression by Upstream Stimulatory Factor. Blood 108 (2006) 4237-4245.

[21] G. Williamson, G.W. Plumb, Y. Uda, K.R. Price, M.J.C. Rhodes. Dietary quercetin glycosides: antioxidant activity and induction of the anticarcinogenc phase II marker enzyme quinone reductase in Hepa1c1c7 cells. Carcinogenesis 17 (1996) 2385-2387.

[22] D.J. Long II, A. Gaikwad, A. Multani, S. Pathak, C.A. Montgomery, F.J. Gonzalez, A.K. Jaiswal. Disruption of the NAD(P)H:quinone oxidoreductase 1 (NQO1) gene in mice causes myelogenous hyperplasia. Cancer Res. 62 (2002) 3030-3036.

[23] J.L. Moran, D. Siegel, D. Ross. A potential mechanism underlying the increased susceptibility of individuals with a polymorphism in $\mathrm{NAD}(\mathrm{P}) \mathrm{H}$ :quinone oxidoreductase 1 (NQO1) to benzene toxicity. Proc. Natl. Acad. Sci. USA 96 (1999) 8150-8155. 
[24] E. Sergediene, K. Jönsson, H. Szymusiak, B. Tyrakowska, I.M.C.M. Rietjens, N. Cenas. Prooxidant toxicity of polyphenolic antioxidants to HL-60 cells: description of quantitative structure-activity relationships. FEBS Lett. 462 (1999) 392-396.

[25] K. Itoh, N. Wakabayashi, Y. Katoh, T. Ishii, K. Igarashi, J.D. Engel, M. Yamamoto. Keap1 represses nuclear activation of antioxidant responsive elements by $\mathrm{Nrf} 2$ through binding to the amino-terminal Neh2 domain. Genes Dev. 13 (1999) 76-86.

[26] B. Catalgol, S. Batirel, Y. Taga, N.K. Ozer. Resveratrol: French paradox revisited. Front Pharmacol. 3 (2012) 141. doi.org/10.3389/fphar.2012.00141

[27] P. Kopp. Resveratrol, a phytoestrogen found in red wine. A possible explanation for the conundrum of the 'French paradox'? Eur. J. Endocrinol. 138 (1998) 619-620 


\section{Figures and Table legends}

\section{Table 1}

List of polyphenols used in this study. All were used in vitro but where indicated the dosages of compounds used in vivo are shown.

Fig.1. Screening of dietary polyphenols for Nrf2 pathway activation. (A) Schematic of plasmid reporter constructs. The human $Q R 1$ promoter hQR1Pluc (Wt and ARE mutant constructs); the transcription start site $(+1)$ and nucleotide positioning is based on ref. 23. Mutations within the TGAC and GC boxes are shown. A putative AP1 site within the ARE is underlined. Lower panel: Enhancer constructs derived from the AREs of $Q R I$ and GST-Ya in transcriptional fusions with the minimal SV40 early promoter and luciferase gene to generate QR-AREluc and GST-AREluc respectively. ARE-Mtluc is QR-AREluc with mutations in the TGAC and GC boxes. Arrow indicates direction of transcription. (B). Differential activation of ARE-dependent luciferase expression by polyphenols. HepG2 cells were transfected with QR-ARELuc or GST-ARELuc and treated with $50 \mu \mathrm{M}$ polyphenols including tBHQ. Luciferase activity was normalized to $\beta$ galactosidase levels. pGL3 Promoter vector backbone and ARE-Mtluc were included for comparison. Fold activation is a ratio of luciferase expression from polyphenol-treated verses DMSO-treated cells. Values are averages of 4 independent transfections. (C) ARE-luc and mutant constructs were transfected alone (filled bars) or with Nrf2 expression plasmid (stippled bars) into HepG2 cells. (D) $Q R 1$ regulation by Nrf2 is ARE-dependent. Full-length promoter and its ARE mutant were co-transfected with Nrf2. Fold activation is a ratio of luciferase expression and basal pGL3 Promoter vector activity with and without Nrf2. Data represent means of 3 independent transfections \pm S.E.M. ${ }^{*}, P$ value $<0.05$ 
Fig. 2 Polyphenols induce Nrf2 transcriptional derepression from Keap1. (A) hQR1Pluc was transfected into HepG2 without or with Nrf2 and increasing concentrations of Keap1. Polyphenols prevent Nrf2 target gene-silencing by Keap1. hQR1Pluc was cotransfected with Nrf2 and Keap1; cells were treated with DMSO or polyphenols. (C, D) Transcriptional derepression of Nrf2 by dietary polyphenols. QR-ARELuc and GST-ARELuc were transfected into HepG2 cells alone or in combination with $100 \mathrm{ng}$ Nrf2. Where indicated, Keap1 was co-transfected and treated with $100 \mu \mathrm{M}$ polyphenols. (E) Nrf2 knockdown diminishes inducibility by polyphenols. Hep3B cells expressing short-hairpin Nrf2 RNA were transfected with $100 \mathrm{ng}$ hQR1Pluc and treated with DMSO or polyphenols for $24 \mathrm{~h}$. Luciferase activities were determined for 3 independent replications of the experiment; all values are averages of these replications \pm S.E.M.

Fig. 3 Polyphenols induce Nrf2 binding to the antioxidant response elements in vitro and in vivo. Nuclear extracts from HepG2 cells treated with either DMSO or polyphenols were incubated with (A) GST-Ya ARE or (B) QR-ARE. Lane designations: 1, ARE oligo only or with nuclear extracts from cells treated with: 2, DMSO; 3, kaempferol; 4, naringenin; 5, quercetin; 6, resveratrol; 7, sulforaphane $(20 \mu \mathrm{M}) ; 8,100 \mu \mathrm{M}$ tBHQ; 9, kaempferol-treated nuclear extracts incubated with mutant ARE or 10, with 100-fold molar excess of unlabeled ARE probe Arrow indicates AREnucleoprotein complex. (C, D) ARE-binding specificity. Recombinant MafG or Nrf2 were incubated with ARE alone (lanes 3 and 4 respectively), or with MafG and increasing concentrations of Nrf2. MafG-Nrf2 heterodimers are indicated with arrows. Competitor probes (cold or mutant ARE) were included as indicated. ARE probe alone (lane 1) or purified GST (lane 2) are negative controls. (E) Chromatin immunoprecipitation assay. HepG2 cells were treated with $100 \mu \mathrm{M}$ kaempferol, naringenin, quercetin, and DMSO. Chromatin was immunoprecipitated with anti-Nrf2 antibody or without antibody. A 500 bp fragment of the $Q R 1$ promoter was amplified by PCR from $10 \%$ of the eluted chromatin. Non-immunoprecipitated chromatin was used as (Input) positive control. 
Fig. 4 Dietary polyphenols induce Nrf2 pathway genes. (A). In vivo activation of $Q R 1$ and GST$Y a$ by dietary polyphenols. Two Northern blots of liver RNA isolated from rats fed DMSO, kaempferol, naringenin, quercetin, and resveratrol were probed separately with $Q R 1$ (top panel) or GST-Ya (middle panel) oligonucleotide probes. Bottom panel is an ethidium bromide-stained denaturing agarose gel of ribosomal RNA (rRNA) to ascertain equivalent RNA loading. (B) Western blotting. Rat liver cell nuclear extracts from the above treatments were probed for Nrf2 expression; $\beta$-actin was used as internal control. 


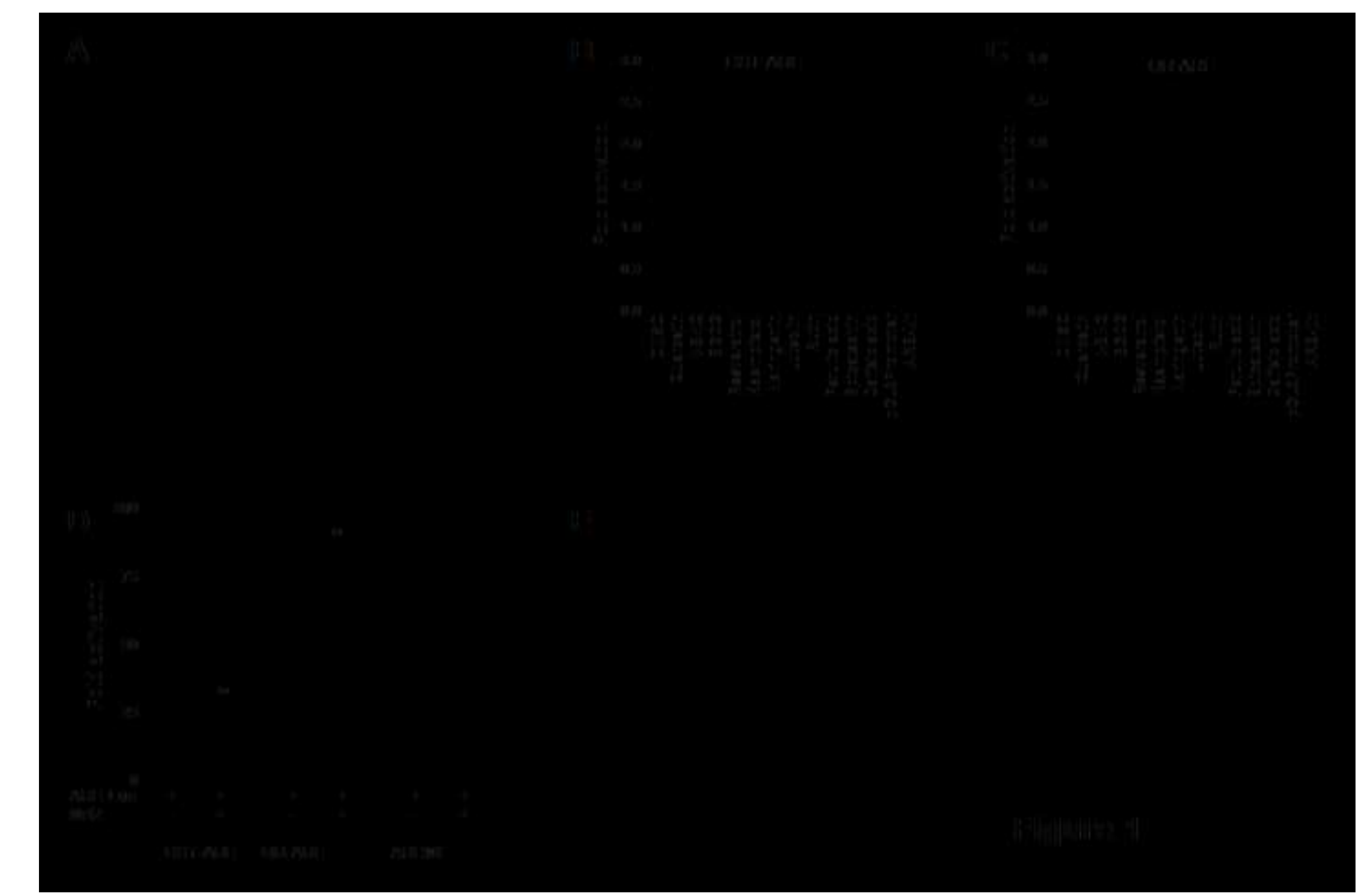

e

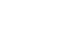
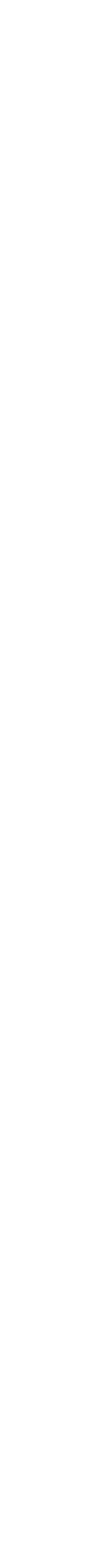
Figure $2 R$
Click here to download high resolution image

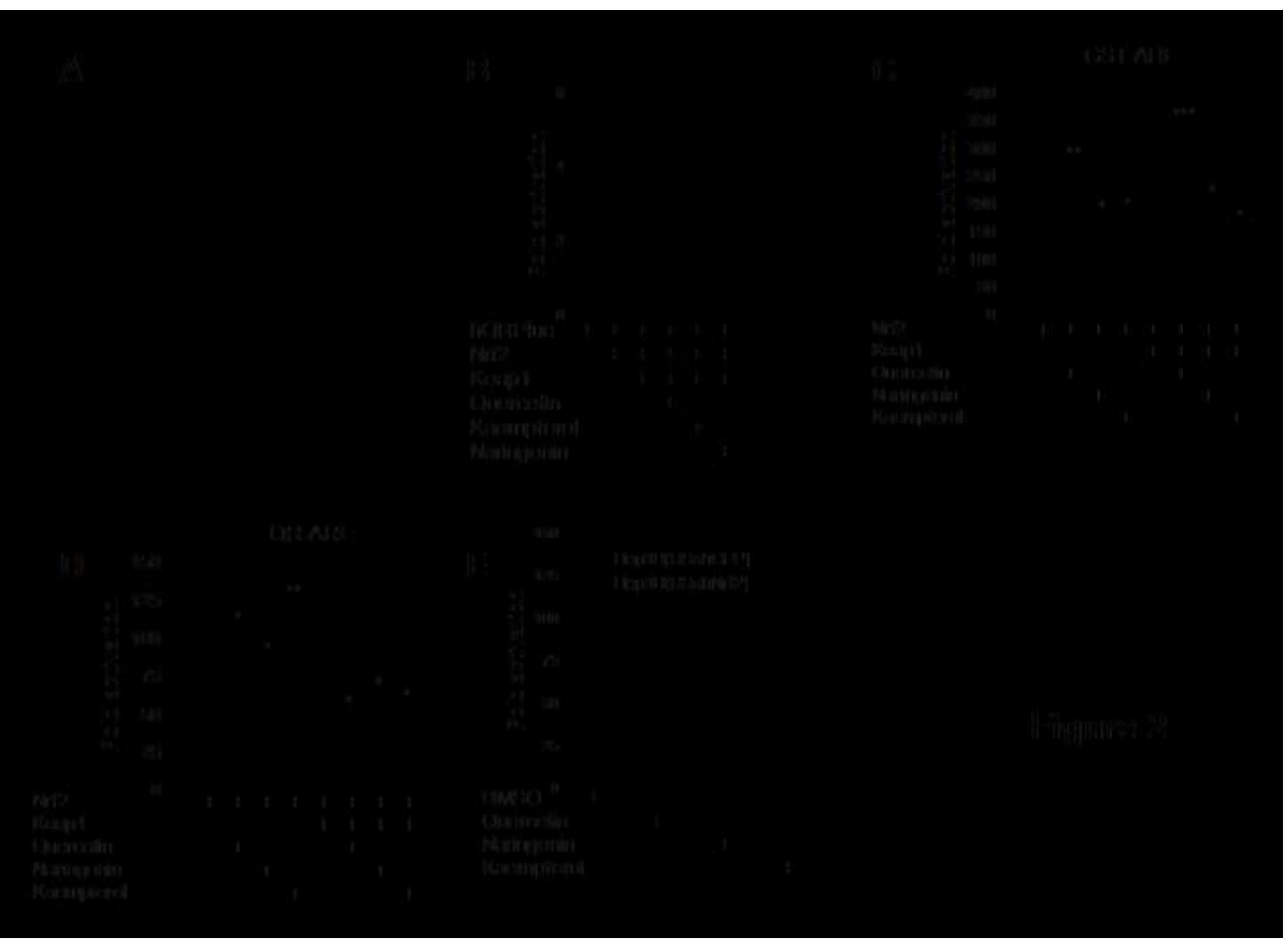


Figure 3R

Click here to download high resolution image

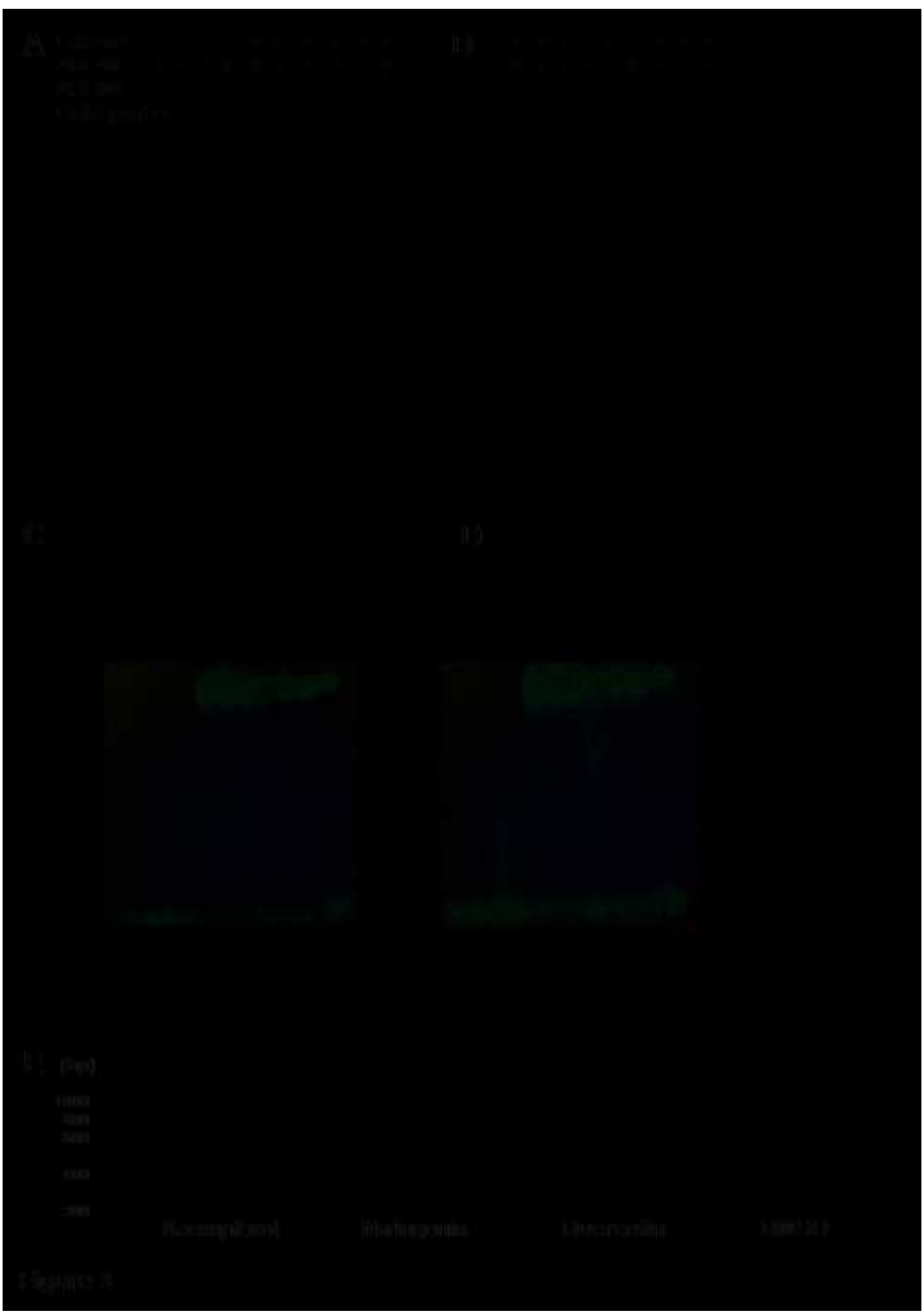



Electronic Supplementary Material (online publication only)
Click here to download Electronic Supplementary Material (

Click here to download Electronic Supplementary Material (online publication only): BBRC-15-4536-Table 1.doc 
*Conflict of Interest
Click here to downloa

Click here to download Conflict of Interest: coi_disclosure-Bayele.pdf

Click here to download Conflict of Interest: coi disclosure-Bayele.pdf 
*Conflict of Interest
Click here to downloa

Click here to download Conflict of Interest: coi_disclosure_Debnam.pdf

- 
Click here to download Conflict of Interest: coi_disclosure-Srai.pdf

${ }^{*}$ Conflict of Interest
Click here to downlo

Click here to downlt 